19 Revue d'histoire du XIXe siècle

Société d'histoire de la révolution de 1848 et des

révolutions du XIXe siècle

35 | 2007

La Restauration revisitée - Les formes de la protestation - Une histoire de l'Etat

\title{
La guerre de Sécession
}

\section{Duncan Andrew Campbell}

Traducteur : Fanja Andriamialisoa

\section{OpenEdition \\ Journals}

Édition électronique

URL : http://journals.openedition.org/rh19/2232

DOI : $10.4000 /$ rh 19.2232

ISSN : $1777-5329$

Éditeur

La Société de 1848

Édition imprimée

Date de publication : 20 décembre 2007

Pagination : 141-159

ISSN : 1265-1354

Référence électronique

Duncan Andrew Campbell, « La guerre de Sécession », Revue d'histoire du XIXe siècle [En ligne], 35 |

2007, mis en ligne le 20 décembre 2009, consulté le 10 décembre 2020. URL : http://

journals.openedition.org/rh19/2232 ; DOI : https://doi.org/10.4000/rh19.2232 


\section{DUNCAN ANDREW CAMPBELL}

\section{La guerre de Sécession}

La guerre de Sécession (1861-1865) fut le conflit le plus sanglant du monde occidental entre les guerres napoléoniennes et la Première Guerre mondiale. Les hostilités furent déclenchées lorsque onze États du Sud entamèrent un processus de sécession des États-Unis pour former les États confédérés d'Amérique, présidés par Jefferson Davis. Le gouvernement fédéral, dirigé par le président Abraham Lincoln, déclara cette sécession illégale et engagea la guerre contre ces États. Après la perte de près de 620000 soldats des deux côtés (les pertes civiles étaient encore plus importantes), la guerre se solda par la victoire des États-Unis (le Nord) contre les États sécessionnistes (le Sud). Pendant la période dite de «reconstruction", ces États du Sud furent tous politiquement réintégrés dans l'Union et regagnèrent dès 1877 une gouvernance autonome au sein de la structure fédérale. Cette guerre laissa des séquelles importantes, encore ressenties aujourd'hui. Le statut actuel des États-Unis comme première puissance mondiale, l'étendue de l'autorité du gouvernement fédéral, les problèmes des relations raciales aux États-Unis ainsi que la récente réémergence de la force et de l'influence politiques des États du Sud sont, dans une certaine mesure, des éléments hérités de ce conflit.

Feu Shelby Foote, l'un des principaux spécialistes de la guerre de Sécession, l'appelait le "carrefour de notre devenir [celui de l'Amérique].» Un autre historien éminent, James McPherson, la décrit comme «la guerre qui ne disparaittra jamais ». La plupart des livres d'histoire divisent celle des États-Unis en deux périodes : la première commence en 1492 et s'achève à la fin de la Reconstruction; la seconde partie va de cette époque à nos jours. De nombreux termes alternatifs ont été utilisés pour désigner ce conflit : pendant le conflit et plusieurs années après, l'Union l'appelait la "Guerre de rébellion»; les États sudistes défaits la nommaient la "Guerre entre les États» et la "Guerre d'indépendance du Sud». Aujourd'hui, le terme de "civil war» est communément utilisé par les spécialistes. Ces dernières années, des États du Sud (le plus récent étant le Mississipi) ont organisé des référendums pour décider d'un retrait éventuel des emblè- 
mes confédérés sur les drapeaux de l'État; des protestations se sont également élevées contre l'érection d'une statue de Lincoln à Richmond en Virginie (l'ancienne capitale des États confédérés). Le débat sur les causes de la guerre se poursuit : fut-elle principalement causée par l'attachement du Sud à l'esclavage, ou par la doctrine affirmant que l'autorité politique ultime appartenait aux États et non au gouvernement fédéral? Il est certain que les États confédérés avaient tous maintenu l'esclavage, alors que les États restés dans l'Union l'avaient aboli. Plus encore, même si ce n'était pas le cas autrefois, les spécialistes s'accordent désormais à imputer la guerre principalement à l'institution de l'esclavage. Il est certain également que les pouvoirs du gouvernement fédéral ont certainement été considérablement renforcés par le conflit. Le terme "États-Unis» n'a commencé à être utilisé au singulier, en anglais, qu'après la victoire de l'Union en 1865. Comme l'observait l'historien Richard Bensel en 1991, l'étude de l'histoire des Etats-Unis devrait débuter en 1865, avec la fin de la guerre de Sécession, bien plus qu'avec la Constitution de $1787^{1}$.

Le moins qu'on puisse dire est que beaucoup d'encre a coulé sur cette guerre. Un inventaire récent a permis de recenser plus de 50000 livres et opuscules publiés sur le sujet, sans compter les dissertations et les articles. Un essai comme celui-ci ne peut que survoler la somme des connaissances sur le sujet et, au mieux, fournir une bonne introduction à certains des débats les plus importants. À l'époque du conflit, les Américains avancèrent tout un tas de raisons justifiant leur engagement dans la guerre, non sans contradictions : après avoir fixé l'émancipation comme objectif de la guerre, Lincoln déclara que l'esclavage était "d'une manière ou d'une autre» la cause de la guerre. Plusieurs sudistes, notamment Davis, récusèrent cette affirmation au moment de la guerre et plusieurs années après. En 1913, l'éminent historien américain James Ford Rhodes déclara qu' «en ce qui concerne la guerre de Sécession, on peut affirmer de manière sure que l'esclavage en était l'unique raison». Cependant, dans les années 1920, les historiens américains de «l'École progressiste» menée par Charles A. Beard affirmèrent que le désaccord entre les groupes d'intérêts et entre les classes était en réalité le facteur déterminant de la guerre. Ils présentèrent le conflit comme une lutte entre une société agraire (le Sud) et une société industrialisée (le Nord). Cette école fut suivie dans les années 1940 par celle des révisionnistes, dominée par Avery Craven et James Randall, qui réfutèrent l'idée de différences profondes entre le Nord et le Sud du pays; ces historiens accusaient plutôt les extrémistes des deux bords - les activis-

1. Cité dans Geoffrey C. Ward, Ric Burns et Ken Burns, The Civil War: An Illustrated History of the War Between the States, London, Pimlico, 1998, p. xvi; James M. McPherson, Drawn with the Sword: Reflections on the American Civil War, Oxford, Oxford University Press, 1996, introduction, et Richard Bensel, Yankee Leviathan: The Origins of Central State Authority in America, 1859-1877, Cambridge, Cambridge University Press, 1990. 
tes contre l'esclavage (les abolitionnistes) et les sécessionnistes du Sud (les "Mangeurs de feu») - d'avoir déclenché une guerre que le reste du pays aurait pu éviter. Dans les années 1950, et plus encore dans les années 1960 avec l'avènement du mouvement des droits civiques, la question ethnique - et donc celle de l'esclavage - domina le débat historiographique sur le conflit. Il semble que la boucle soit ainsi bouclée et que la plupart des historiens considèrent aujourd'hui l'esclavage comme la cause profonde de la guerre. Ceci dit, la majorité des historiens reconnaissent également que la doctrine sur les droits des États était importante pour galvaniser les citoyens et les soldats de la Confédération (parmi ces derniers, moins de $25 \%$ possédaient des esclaves) et que le nationalisme qui prévalait à la moitié du était un élément moteur des activités de l'Union (la plupart des nordistes ne prônèrent l'abolitionnisme qu'après la fin de la guerre) ${ }^{2}$.

Une discussion sur le sujet encore controversé des causes de la guerre nécessite un examen des questions auxquelles était confrontée l'Amérique d'avant la guerre, notamment sur l'équilibre du pouvoir entre les États et le gouvernement fédéral, et même sur la question de savoir si le droit de sécession existait ou non. Ce point n'était effectivement pas clair. En 1839 par exemple, l'ancien président John Quincy Adams déclara publiquement que «si le jour devait venir [...] où les affections des peuples de ces États devaient se désunir [...] il serait beaucoup mieux pour les peuples des États désunis de se séparer dans l'amitié que d'être contraints de demeurer ensemble». Précédemment encore, en 1812, lors de la deuxième guerre opposant les États-Unis à la Grande-Bretagne, les États de la Nouvelle Angleterre, opposés au conflit, menacèrent de se retirer de l'Union si le conflit n'était pas résolu. Mais la première manifestation de sécession sudiste - et de résistance à son égard - date de 1830, lorsque la Caroline du Sud menaça de défier, ou d'annuler (nullify) les droits de douane fédéraux appliqués aux importations européennes afin de protéger les industries naissantes du nord-est des États-Unis. Après que le président Andrew Jackson l'eut menacée d'une action militaire, la Caroline du Sud menaça de faire séces-

2. James Ford Rhodes et Charles A. Beard cités dans Alan Farmer, The Origins of the American Civil War, 1846-1861, London, Hodder \& Stoughton, 1996, p. 145-147. Pour une discussion historiographique sur les causes de la guerre de Sécession, voir Gerald N. Grob et George Athan Billias (eds.), Interpretations of American History : Patterns and Perspectives, troisième édition, New York, Free Press, 1978, volume 1, p. 363-377, et James M. McPherson, "What Caused the Civil War?", North and South, volume IV, $\mathrm{n}^{\circ}$ 1, 2000, p. 12-22. Les meilleures études approfondies sur le conflit sont : Ludwell H. Johnson, North Against South : The American Iliad, 1848-1877, Columbia, S.C., Foundation for American Education, 1995; Richard H. Sewell, A House Divided: Sectionalism and Civil War, 18481865, Baltimore, Johns Hopkins University Press, 1988; James M. McPherson, Battle Cry of Freedom : The Civil War Era, Oxford, Oxford University Press, 1988; Peter J. Parish, The American Civil War, New York, Holmes \& Meier Publishers, 1975; Shelby Foote, The Civil War : A Narrative, 3 volumes, New York, Random House, 1958-1974; Bruce Catton, The Civil War, New York, American Heritage Press, 1971; et Allan Nevins, The War for the Union, 4 volumes, New York, Scribner, 1959-1971. Les études de Johnson et de Foote, qui représentent l'opinion des minorités, soulignent les droits des États plutôt que l'esclavage comme cause de la guerre. 
sion. Cette crise, connue sous le nom de Nullification Crisis, s'acheva par un compromis politique pacifique : les taxes controversées furent diminuées, et la Caroline du Sud retira l'ordonnance d'annulation. En d'autres termes, le litige sur la question de l'autorité fédérale ou de celle des États précédait la querelle sur l'esclavage. Une minorité d'historiens considèrent cet incident comme étant le premier signe des événements à venir ${ }^{3}$.

Toutefois, la Nullification Crisis mit également au jour les profondes divisions économiques entre les États. Ceux du Nord-Est s’industrialisaient rapidement et cette industrialisation, notamment l'émergence du chemin de fer, commençait à transformer l'économie américaine. Le protectionnisme devenait une politique de plus en plus populaire dans ces États industrialisés, qui souhaitaient une fermeture des États-Unis aux produits manufacturés importés de l'étranger. Les économies de l'Ouest et du Sud étaient basées sur les exportations, blé et autres céréales pour l'Ouest et coton pour le Sud : ces États étaient en faveur du libre-échange. Ils voulaient avoir accès aux produits manufacturés européens, moins onéreux et souvent de meilleure qualité, et, pour pouvoir continuer à les acheter, ils avaient besoin d'exporter librement leurs récoltes. Le protectionnisme et le libre-échange devinrent ainsi aux États-Unis des doctrines associées aux positionnements des partis politiques ainsi qu'à des groupes d'intérêt bien identifiés ${ }^{4}$.

Ceci dit, la principale culture d'exportation des États sudistes était le coton, qui était produit grâce au travail des esclaves. L'invention en 1793 de la première égreneuse (qui séparait la fibre de manière peu onéreuse et efficace) rendit la production cotonnière très lucrative. Même si l'imagerie populaire représente un Sud de grands manoirs et de milliers d'esclaves, un

3. La Nullification Crisis a été traitée de manière intelligente dans William W. Freehling, Prelude to Civil War: The Nullification Controversy in South Carolina, 1816-1830 (New York, Harper \& Row, 1966) et Richard Ellis, The Union at Risk: Jacksonian Democracy, States Rights and the Nullification Crisis (New York, Oxford University Press, 1987). Le principal opposant à Jackson lors de cette crise, son ancien vice-président John C. Calhoun, généralement perçu comme le principal porte-parole des droits des États, fait l'objet d'un recueil intéressant : Ross M. Lence (ed.), Union and Liberty : The Political Philosophy of John C. Calhoun, Indianapolis, Liberty Fund, 1992. Un ouvrage présente favorablement le pedigree politique et intellectuel des droits des États, celui de Forrest McDonald, States'Rights and the Union: Imperium in Imperio, 1776-1876, Lawrence, University of Kansas Press, 2000.

4. L'importance croissante de l'industrialisation américaine et les questions connexes telles que le développement économique, le transport, l'urbanisation et le protectionnisme avant la guerre sont examinées par Charles G. Sellars, The Market Revolution : Jacksonian America 1815-1846, Oxford, Oxford University Press, 1991; Thomas C. Cochran, Frontiers of Change : Early Industrialism in America New York, Oxford University Press, 1981 ; Peter Temin, The Jacksonian Economy, New York, Norton, 1969; Albert Fishlow, American Railroads and the Transformation of the Antebellum Economy, Cambridge, Harvard University Press, 1965; Douglass C. North, The Economic Growth of the United States, 1790-1860, Englewood Cliffs (New Jersey), Prentice-Hall, 1961 et George R. Taylor, The Transportation Revolution, 1815-1860, New York, Rinehart, 1951. Cependant, la société américaine était en très grande majorité agraire (la plupart des américains ne vivaient pas dans les zones urbaines avant le début du $\mathrm{Xx}^{\mathrm{e}}$ siècle) et les dimensions agraires des aspects évoqués supra sont présentées dans Steven Hahn et Jonathan Prude, (eds), The Countryside in the Age of Capitalist Transformation : Essays in the Social History of Rural America, Chapel Hill, University of North Carolina Press, 1985 et Paul W. Gates, The Farmers' Age : Agriculture 1815-1860, New York, Holt, Rinehart et Winston, 1960. 
quart des propriétaires d'esclaves ne possédait qu'entre un et neuf esclaves, une bonne moitié en possédait entre dix et 49 et seul un quart en possédait plus de 50. De plus, le nombre de propriétaires d'esclaves déclinait. En 1850, 36\% des sudistes blancs possédaient des esclaves; en 1860, ils étaient moins de $25 \%$. Il faut également noter que si les États du Nord abolirent l'esclavage les uns après les autres, peu d'entre eux accordèrent le droit de citoyenneté aux Noirs américains; le droit de vote, celui de faire partie d'un jury ou d'épouser des Blancs leur étaient refusés. Dans les États de l'Ouest, les dénommés "codes noirs», qui interdisaient aux Noirs américains d'y vivre ou d'y déménager, étaient également populaires. La majorité des historiens reconnaissent que ce qui fut appelé la «négrophobie» dominait de manière écrasante l'opinion des Américains blancs, que ce soit au Nord ou au Sud 5 .

L'abolitionnisme constituait donc l'exception aux États-Unis. Le mouvement démarra pour soutenir la compensation de l'émancipation (c'està-dire un remboursement des propriétaires qui avaient rendu la liberté à leurs esclaves) et la "colonisation" (la déportation des Noirs américains vers l'Afrique ou vers l'Amérique latine). Dans les années 1830, le mouvement développa une aile radicale - représentée de manière très compétente par son principal porte-parole, William Lloyd Garrison, et son journal The Liberator - qui exigeait une émancipation immédiate et non compensée des esclaves. Ce mouvement, principalement composé de chrétiens évangéliques et tirant sa force des États de la Nouvelle-Angleterre, suscita en retour dans le Sud un attachement affiché à l'esclavage, ce qui éloignait toute possibilité de compromis sur la question de l'abolitionnisme. Si les abolitionnistes étaient autrefois traités de fanatiques, les historiens louent aujourd'hui leur courage moral - en particulier celui de l'aile radicale pour avoir osé défier l'institution de l'esclavage ${ }^{6}$.

5. L'esclavage américain a fait l'objet de presque autant d'ouvrages que la guerre elle-même, mais les introductions de qualité sur le sujet sont Peter Kolchin, American Slavery, 1619-1877, New York, Hill et Wang, 1993 et Louis Filler, Slavery in the United States of America, New York, Van Nostrand, 1972. Pour le sujet moins étudié du traitement réservé aux noirs dans les États du Nord, par opposition aux États du Sud, voir Leon Litwack, North of Slavery: The Negro in the Free States, 1790-1860, Chicago, University of Chicago Press, 1961 et Eugene H. Berwanger, The Frontier Against Slavery: Western Anti-Negro Prejudice and the Slavery Extension Controversy, Urbana, University of Illinois Press, 1967. En contraste, le sujet de l'esclavage sudiste et les réactions dans le reste du pays ont fait l'objet d'un tas d'études, notamment, Robert W. Fogel, Without Consent or Contract: The Rise and Fall of American Slavery, New York, Norton, 1989; Eugene Genovese, Roll Jordan, Roll: The World the Slaves Made, New York, Pantheon Books, 1974; Robert W. Fogel et Stanley L. Engerman, Time on the Cross : The Economics of American Negro Slavery, Boston, Little and Brown, 1974; John W. Blassingame, The Slave Community : Plantation Life in the Antebellum South, New York, Oxford University Press, 1972; Stanley M. Elkins, Slavery : A Problem in American Institutional and Intellectual Life, Chicago, Chicago University Press, 1959 et Kenneth M. Stampp, The Peculiar Institution: Slavery in the Ante-bellum South, New York, Knopf, 1956.

6. Sur les opposants à l'esclavage, voir Herbert Aptheker, Abolitionism : A Revolutionary Movement, Boston, Twayne Publishers, 1989; James Brewer Stewart, Holy Warriors : The Abolitionists and American Slavery, New York, Hill and Wang, 1976; et Merton L. Dillon, The Abolitionists : The Growth of a Dissenting Minority, DeKalb, Northern Illinois University Press, 1974. Un ouvrage à ne pas négliger 
L'expansion des États-Unis vers l'Ouest expliquait l'importance des tarifs douaniers et de l'esclavage pour le pays dans son ensemble comme pour les différents groupes d'intérêt. Cette expansion suscite actuellement de plus en plus d'intérêt comme facteur de déclenchant de la guerre. Alors que les pionniers américains traversaient le continent vers l'Ouest et en déplaçaient les indigènes, l'équilibre entre les États du Nord et du Sud était préservé par le "compromis du Missouri» de 1819, qui prohibait l'esclavage dans le territoire de la Louisiane au nord de la latitude $36^{\circ} 30^{\prime}$. Par la suite, le compromis tomba à l'eau car si l'expansionnisme du Nord s'arrêta aux frontières de ce qui est maintenant le Canada, - territoire alors sous l'autorité de l'Empire britannique -, l'expansionnisme du Sud se poursuivit sans entraves jusque dans le territoire mexicain. L'admission de la République du Texas (qui fit sécession du Mexique en 1835-1836) en tant qu'État dans l'Union fut retardée par plusieurs États du nord-est, qui craignaient de se voir dépassés en nombre par les États sudistes. Ainsi, en 1844, Joshua Giddings, le représentant de l'Ohio au Congrès, accusa le Sud de chercher par l'expansion territoriale à augmenter le nombre d'États sudistes pour obtenir plus de soutien pour le libre-échange, plutôt que pour préserver l'esclavage. Cependant, c'est avec l'expansion territoriale américaine vers l'Ouest (appelée plus tard la "destinée manifeste» ou Manifest Destiny), qui culmina avec la guerre contre le Mexique en 1846-1848 au cours de laquelle les États-Unis annexèrent plus de la moitié du territoire mexicain, que se révélèrent les différends entre Nord et Sud. Les tentatives de représentants au Congrès des États du Nord et de l'Ouest de déclarer tout territoire mexicain annexé "terres libres» (en anglais, free soil, c'està-dire dépourvu de Noirs, qu'ils soient esclaves ou libres) mirent au jour durablement ces dissensions. Seul le compromis de 1850, qui divisa le territoire en États libres et en États esclavagistes, ainsi que l'introduction de la loi sur les esclaves fugitifs, qui obligeait le gouvernement fédéral à capturer et à renvoyer les esclaves fugitifs, calmèrent temporairement les passions des différentes partis. Les études récentes sur la guerre de Sécession considèrent que la guerre du Mexique renforça irrémédiablement la division entre les États nordistes et sudistes ${ }^{7}$.

est la récente biographie de John Brown, l'abolitionniste militant dont l'initiative avortée en faveur d'un soulèvement d'esclaves en Virginie en 1859 avait permis de placer l'esclavage au cËur de lélection présidentielle de 1860, avec pour résultat l'élection de Lincoln et la sécession de la Caroline du Sud : David S. Reynolds, John Brown, Abolitionist : The Man Who Killed Slavery, Sparked the Civil War and Seeded Civil Rights, New York, Knopf, 2005. Voir également Stanley Harrold, The Abolitionists and the South, 1831-1861, Lexington, University Press of Kentucky, 1995 et Judith Edwards, Abolitionists and Slave Resistance : Breaking the Chains of Slavery, Berkeley Heights (New Jersey), Enslow Publishers, 2004.

7. Pour une introduction sur ce sujet, voir Michael A. Morrison, Slavery and the American West: The Eclipse of Manifest Destiny and the Coming of the Civil War, Chapel Hill, University of North Carolina Press, 1997; Anders Stephanson, Manifest Destiny : American Expansionism and the Empire of Right, New York, Hill and Wang, 1995; William W. Freehling, The Road to Disunion, New York, Oxford University Press, 1990; Thomas R. Hietala, Manifest Design : Anxious Aggrandizement in Late 
La décennie 1850 fut extrêmement volatile : la société américaine connut des changements culturels majeurs, liés notamment à l'immigration de masse; elle était divisée en États esclavagistes et États libres; certains États bénéficiaient d'une urbanisation et d'une industrialisation rapides tandis que d'autres se faisaient défi de demeurer ruraux. Les querelles, les débats, les désaccords étaient nombreux : fallait-il préférer le libre échange au protectionnisme? La souveraineté ultime appartenait-elle au gouvernement fédéral ou aux États? Voire, les États-Unis étaient-ils une union ou une nation? Le Parti national whig, incapable de répondre à ces nouveaux défis, s'effondra - il serait ultérieurement remplacé par le Parti fractionnel républicain. À leur tour, les Républicains ne réussirent qu'à empêcher les Know Nothing ou l'American Party, un mouvement anti-immigrant et nativiste, d'usurper leur place d'opposant au Parti démocrate. L'effondrement du système bipartite traditionnel, car les Whigs et les Démocrates avaient tous les deux des représentants au Nord et au Sud, signifiait que les partis politiques étaient également fractionnés et était un signe avant-coureur de la collision à venir ${ }^{8}$.

Trois sujets spécifiques agitèrent les années 1850 : la sévère récession économique de 1857, le statut d'admission (État libre ou esclavagiste) du territoire du Kansas dans l'Union et enfin la décision de la Cour suprême sur l'affaire Dred Scott en 1856. Cette affaire commença lorsque l'esclave Scott tenta de gagner sa liberté en plaidant qu'il vivait maintenant dans un État libre et était, par conséquent, libre. Le verdict de cette affaire devait déterminer à la fois la légitimité de l'Acte fédéral sur les esclaves fugitifs et le compromis politique qui permettait aux États-Unis d'être divisé en États libres et esclavagistes. La Cour se prononça contre Scott et mit fin au compromis qui permettait aux États libres d'empêcher l'importation de l'esclavage sur leur territoire. En déclarant que les hommes blancs n'avaient à respecter aucun droit des hommes noirs, la Cour décrétait que les esclaves pourraient être emmenés n’importe où aux États-Unis. Par conséquent, les propriétaires sudistes d'esclaves obtenaient tout ce qu'ils voulaient, notam-

\footnotetext{
Jacksonian America, Ithaca (New York), Cornell University Press, 1985; Don E. Fehrenbacher, Manifest Destiny and the Coming of the Civil War, 1840-1861, New York, Appleton-Century-Crofts, 1970 et Ray A. Billington, Far Western Frontier, 1830-1860, New York, Harper, 1956.

8. La rupture du système bipartite est examinée de manière experte dans l'étude magistrale de Michael F. Holt, The Rise and Fall of the American Whig Party : Jacksonian Politics and the Onset of the Civil War, New York, Oxford University Press, 1999, qui explore plus que le titre ne le suggère. Une autre étude de qualité par le même auteur est The Political Crisis of the 1850s, New York, Wiley, 1978. Sur la montée du nouveau Parti républicain, voir Eric Foner, Free Soil, Free Labor, Free Men: The Ideology of the Republican Party Before the Civil War, New York, Oxford University Press, 1970 et William E. Gienapp, The Origins of the Republican Party, 1852-1856, New York, Oxford University Press, 1987. Sur les "Know-Nothing" voir Tyler Anbinder, Nativism and Slavery: The Northern Know Nothings and the Politics of the 1850s, New York, Oxford University Press, 1992. Sur les divisions et les difficultés du Parti démocrate, voir Roy F. Nichols, The Disruption of the American Democracy, New York, Macmillan Co., 1948 qui est une étude plus ancienne mais encore très utile.
} 
ment l'expansion illimitée de leur institution. L'hostilité entre les partis atteignit clairement un point de non-retour.

La controverse sur le Kansas-Nebraska portait sur le statut d'admission, en tant qu'État libre ou État esclavagiste, de ce territoire dans l'Union. Deux capitales rivales furent établies dans le territoire; chacune rédigea une nouvelle constitution, l'une interdisant l'esclavage et l'autre l'autorisant. La violence éclata à ce moment-là entre les deux groupes, suspendant ainsi le processus d'admission dans l'Union. Bien que la plupart des historiens actuels minimisent l'importance de l'affaire Dred Scott et la violence au Kansas-Nebraska, - mettant plutôt l'accent sur la récession de 1857 (qui fit plus de tort aux États industriels qu'à ceux qui dépendaient de l'agriculture) -, les deux événements contribuèrent néanmoins à la détérioration de la situation politique ${ }^{9}$.

Lors de l'élection de 1860, les Démocrates se divisèrent et proposèrent deux candidats à la présidence. Grâce à cet éclatement de l'opposition, Lincoln, le candidat du Parti républicain, gagna l'élection le 6 novembre 1860 à la majorité relative. Pas un seul État du Sud ne lui apporta ses suffrages dans le collège électoral. Le Sud rejeta en bloc la plateforme politique totalement nordiste du Parti républicain, qui impliquait des taxes douanières plus élevées, une augmentation des fonds fédéraux consacrés au développement industriel et l'admission du Kansas en tant qu'État libre dans l'Union. La législature de l'État de la Caroline du Sud vota à l'unanimité pour l'indépendance et l'État fit sécession de l'Union; la Caroline du Sud fut rejointe plus tard par d'autres États de la partie inférieure du Sud, notamment, l'Alabama, le Mississipi, la Géorgie, la Louisiane, la Floride et le Texas. Le 4 février 1861, ces États formèrent une nouvelle union, les États confédérés d'Amérique, dirigés par un président provisoire, Jefferson Davis. Plusieurs compromis politiques furent proposés (notamment un amendement constitutionnel garantissant que le gouvernement fédéral

9. Sur le Kansas-Nebraska voir Gunja S. Gupta, For God and Mammon : Evangelicals and Entrepreneurs, Masters and Slaves in Territorial Kansas, Athens, University of Georgia Press, 1996 et James A. Rawley, Race \& Politics: 'Bleeding Kansas' and the Coming of the Civil War, Philadelphia, Lippincott, 1969. Sur la décision de l'affaire Dred Scott, voir D. J. Herda, The Dred Scott Case : Slavery and Citizenship, Hillside, (New Jersey), Enslow Publishers, 1994 et Don E. Fehrenbacher, The Dred Scott Case : Its Significance in American Law and Politics, New York, Oxford University Press, 1978. Sur les tentatives de compromis pour l'extension du territoire esclavagiste entre les États esclavagistes et non esclavagistes, ainsi que sur les personnalités impliquées, voir John Ashworth, Slavery, Capitalism and Politics in the Antebellum Republic, volume 1, Commerce and Compromise, 1820-1850, Cambridge, Cambridge University Press, 1995; Bruce Levine, Half Slave and Half Free: The Roots of the Civil War, New York, Hill and Wang, 1992; Peter Knupfer, The Union as It Is : Constitutional Unionism and Sectional Compromise, 1787-1861, Chapel Hill, University of North Carolina Press, 1991; Merrill D. Peterson, The Great Triumvirate: Webster, Clay and Calhoun, New York, Oxford University Press, 1987; Paul Finkleman, An Imperfect Union: Slavery, Federalism and Comity, Chapel Hill, University of North Carolina Press, 1981. Pour avoir un bon aperçu des crises politiques dans le pays dans les années 1850, notamment la récession, voir James L. Huston, The Panic of 1857 and the Coming of the Civil War, Baton Rouge, Louisiana State University Press, 1987 et Kenneth Stampp, America in 1857 : A Nation on the Brink, New York, Oxford University Press, 1990) 
n'abolirait pas l'esclavage) mais furent rejetés par la nouvelle Confédération. Si le président sortant James Buchanan déclara la sécession illégale, il affirma néanmoins que le gouvernement fédéral n’avait pas le droit de recourir à des voies militaires pour l'empêcher. Par conséquent, il n'entreprit rien tandis que les États du Sud s'organisèrent. Le président nouvellement élu, Lincoln, exprima son accord avec la première partie de la déclaration de Buchanan mais déclara que la guerre restait une option. La guerre était inévitable : le reste des États-Unis n'accepterait pas l'indépendance des États du Sud et ces derniers ne rejoindraient pas volontairement l'Union ${ }^{10}$.

L'événement qui déclencha l'engagement militaire fut l'attaque de l'armée de la Confédération sur le fort fédéral Sumter, gardé par des soldats américains qui refusèrent de se rendre aux sécessionnistes. Lincoln, qui avait essayé de trouver une solution pacifique à la crise, fit appel à 75000 hommes pour supprimer une révolution «trop puissante pour pouvoir être supprimée par le cours ordinaire des procédés judiciaires " ${ }^{11}$ et ordonna le blocus du Sud. Les États de la partie septentrionale du Sud, comprenant la Virginie, l'Arkansas, la Caroline du Nord et le Tennessee, opposés aux mesures coercitives contre les États confédérés, rompirent avec l'Union et rejoignirent la nouvelle Confédération, qui avait pour capitale Richmond en Virginie. Davis fit appel à 100000 hommes pour défendre la nouvelle nation. Il y avait maintenant deux communautés politiques distinctes disposant d'un gouvernement autonome et d'une armée pour soutenir leurs causes respectives ${ }^{12}$.

10. Sur la crise sécessionniste elle-même, au Nord comme au Sud, les meilleures études sont : Donald E. Reynolds, Editors Make War : Southern Newspapers in the Secessionist Crisis, Nashville, Vanderbilt University Press, 1970; Ralph Wooster, The Secession Conventions of the South, New Jersey, Princeton, 1962; et Kenneth Stampp, And the War Came: The North and the Secessionist Crisis, 18601861, Baton Rouge, Louisiana State University Press, 1950. Sur la position du président Buchanan, voir Elbert B. Smith, The Presidency of James Buchanan, Lawrence, University of Kansas Press, 1975. Sur celle de son successeur, voir Don E. Fehrenbacher, Prelude to Greatness : Lincoln in the 1850s, Stanford, Stanford University Press, 1962 et David M. Potter, Lincoln and His Party in the Secession Crisis, New Haven (Connecticut), Yale University Press, 1962. Il faut explorer la société du Sud pour comprendre comment le mouvement sécessionniste gagna du terrain. Plusieurs études utiles existent sur ce sujet notamment : Eric H. Walther, The Fire-Eaters, Baton Rouge, Louisiana State University Press, 1992; Bruce Collins, White Society in the Antebellum South, London, Longman, 1985; J Oakes, The Ruling Race : A History of Southern Slaveholders, New York, Knopf, 1982; Bertram Wyatt-Brown, Southern Honor: Ethics and Behaviour in the Old South, New York, Oxford University Press, 1982; John McCardell, The Idea of a Southern Nation: Southern Nationalists and Southern Nationalism, 1830-1860, New York, Norton, 1979; Gavin Wright, The Political Economy of the Cotton South: Households, Markets and Wealth, New York, Norton, 1978 et Frank L. Owsley, Plain Folk of the Old South, Baton Rouge, Louisiana State University Press, 1949.

11. Roy P. Basler (ed.), The Collected Works of Abraham Lincoln, New Brunswisck (New Jersey), Rutgers University Press, 1953-1955, 8 volumes, volume 4, p. 332.

12. Deux études sur l'assaut du fort Sumter sont : Richard N. Current, Lincoln and the First Shot, Philadelphia, Lippincott, 1963 et William A. Swanberg, First Blood: The Story of Fort Sumter, New York, Scribner, 1957. Plusieurs des ouvrages cités ci-dessus décrivent les causes du conflit, mais les travaux utiles qui examinent directement le sujet sont : Brian Holden Reid, The Origins of the American Civil War, London, Longman 1996; Gabor S. Boritt (ed.), Why the Civil War Came, New York, Oxford University Press, 1996; Kenneth M. Stampp, The Causes of the Civil War, troisième édition, New York, 
La bataille elle-même fut engagée sur deux fronts : Est et Ouest. Le front Est peut être décrit comme la région qui s'étend de Washington à Richmond; le front Ouest couvrait le cours supérieur et inférieur du fleuve Mississipi et ensuite, à partir de 1863, de l'est du fleuve à la Caroline du Sud. En d'autres termes, à l'exception de la bataille de Gettysburg en Pennsylvanie en 1863, cette guerre fut menée dans les États sudistes et dans les États frontaliers. Si les historiens de la guerre se sont traditionnellement concentrés sur le front de l'Est, le front de l'Ouest est maintenant perçu comme celui qui détermina l'issue de la guerre. Sur le front de l'Est, le Sud eut d'abord le dessus, venant à bout des armées de l'Union à la première bataille de Bull Run (juillet 1861), aux batailles des sept jours (25 juin-

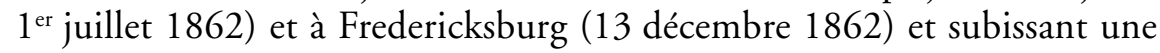
seule défaite majeure à Antietam (17 septembre 1862) lorsqu'il tenta d'envahir le Maryland dans l'espoir de prendre Washington. La situation du front de l'Ouest était différente. L'Union l'emportait, prenant contrôle du territoire au nord du fleuve de Mississipi, une position maintenue après la bataille de Shiloh (6-7 avril), ainsi qu'au sud après la conquête de la Nouvelle Orléans (28 mai 1862). L'Union acheva de contrôler totalement le fleuve Mississipi lorsqu'elle conquit Vicksburg (4 juillet 1863), coupant la Confédération en deux ${ }^{13}$.

C'est après Antietam que Lincoln publia la Proclamation d'émancipation (22 septembre), déclarant qu'au $1^{\text {er }}$ janvier 1863, tous les esclaves dans les États rebelles seraient désormais «libres, maintenant et à jamais». Lincoln agit tardivement contre l'esclavage car sa priorité était de préserver l'Union et non d'émanciper les esclaves. Il était par ailleurs préoccupé par les États frontaliers esclavagistes qui étaient restés loyaux à l'Union, ainsi que par l'opposition considérable à l'émancipation au Nord. Les raisons exactes du choix de Lincoln d'agir à ce moment précis restent controversées, mais les historiens citent plusieurs arguments possibles : le Président était

Simon \& Schuster, 1991; Edwin C. Rozwenc, The Causes of the American Civil War, deuxième édition, Lexington (Massachussets), Heath, 1972 et Hans Trefousse (ed.), The Causes of the Civil War: Institutional Failure or Human Blunder?, New York, Holt, Rinehart and Winston, 1971. Une opinion intéressante, voire singulière, sur les origines de la guerre peut être trouvée dans Charles Adams, When in the Course of Human Events : Arguing the Case for Southern Secession, Lanham (Maryland), Rowman \& Littlefield, 2000, qui avance que les débats sur les taxes douanières, et non l'esclavage, étaient à l'origine de la sécession sudiste.

13. Les études historiques de la guerre sont innombrables et difficiles à recenser correctement. Il serait à peine exagéré de dire que chaque général a son biographe et chaque régiment son historien. Tous les ouvrages généraux cités dans la note 2 constituent un bon point de départ. Ceux cités ci-dessous sont simplement des ouvrages utiles supplémentaires. Sur les activités militaires générales, voir Bell I. Wiley, The Life of Johnny Reb, The Common Soldier of the Confederacy, Indianapolis : Bobbs-Merrill, 1943 et The Life of Billy Yank, The Common Soldier of the Union, Indianapolis, Bobbs-Merrill, 1952; Gerald F. Linderman, Embattled Courage: The Experience of Combat in the American Civil War, New York, Free Press, 1987 et Archer Jones, Civil War Command and Strategy: The Process of Victory and Defeat, New York, Free Press, 1992. Sur Antietam, voir James M. McPherson, Crossroads of Freedom : Antietam, Oxford, Oxford University Press, 2002. Sur New Orleans et Farragut, voir Robert J. Schneller, Farragut: America's First Admiral, Washington, Brassey's, 2002. 
sous la pression de membres pro-abolitionnistes du Congrès; il avait besoin de recruter des Noirs américains dans les rangs de l'armée de l'Union ou il cherchait à décourager une intervention étrangère potentielle dans la guerre (en particulier de la Grande-Bretagne et de la France) en s'alignant sur les positions abolitionnistes de ces nations ${ }^{14}$.

L'émancipation étant maintenant l'un des objectifs de guerre de l'Union, les historiens considèrent désormais l'année 1863 comme le tournant de la guerre pour plusieurs raisons. Si sur le front de l'Est, le Sud gagna la bataille de Chancellorsville (2 mai 1863), sa seconde invasion du Nord se solda par un désastre à Gettysburg en Pennsylvanie ( $1^{\text {er }}-3$ juillet 1863 ). Cette bataille coûta à la Confédération de trop nombreux hommes, qui furent difficiles à remplacer. Par conséquent, Gettysburg et Vicksburg signèrent le début de la fin pour un Sud réduit à une guerre défensive. Cette situation fut confirmée sur le front de l'Ouest par les victoires de l'Union à Chickamauga et à Chattanooga au Tennessee (24-25 novembre 1863) ainsi que par la prise d'Atlanta en Géorgie (3 septembre 1864), et sur le front de l'Est par les batailles de la Wilderness (5-7 mai 1864), de Spotsylvania (8-12 mai 1864) et de Cold Harbor (1-3 juin 1864) ${ }^{15}$.

À la fin de l'année 1864, la défaite du Sud, battu sur les deux fronts, paraissait évidente. Lincoln, réélu président en grande partie grâce à la prise d'Atlanta, déclara clairement qu'il continuerait la guerre jusqu'au bout. Richmond tomba le 3 avril 1865, et à la fin du mois, la plupart des armées sudistes s'étaient rendues. Le vendredi de Pâques 14 avril 1865, Lincoln, au sommet de son triomphe, fut assassiné au Théâtre Ford à Washington par un sympathisant de la cause sudiste, John Wilkes Booth ${ }^{16}$.

Le martyre de Lincoln fut un événement déterminant de la guerre et l'éleva à la sainteté politique. Il devint «le grand émancipateur» et le «sauveur de l'Union ». Il n'est pas étonnant que les historiens le citent régulièrement comme le plus grand président américain de l'histoire, voire comme l'Américain le plus éminent de tous les temps. La réputation de Lincoln n'est pas usurpée. Son discours à Gettysburg, après la bataille, est l'un des grands

14. Sur la Proclamation de l'émancipation, voir John Hope Franklin, The Emancipation Proclamation, Garden City (New York), Doubleday, 1963; Howard Jones, Abraham Lincoln and a New Birth of Freedom: The Union and Slavery in the Diplomacy of the Civil War, Lincoln, University of Nebraska Press, 1999; Allen C. Guelzo, Lincoln's Emancipation Proclamation : The End of Slavery in America, New York, Simon \& Schuster, 2004; Silvana R. Siddali, From Property to Person : Slavery and the Confiscation Acts, 1861-1862, Baton Rouge, Louisiana State University Press, 2005.

15. Sur Chancellorsville, Chickamauga et Chattanooga, voir Stephen W. Sears, Chancellorsville, Boston, Houghton-Mifflin, 1996; Glenn Tucker, Chickamanga : Bloody Battle of the West, Indianapolis, Bobbs-Merrill, 1961 et Peter Cozzens, The Shipwreck of Their Hopes: The Battles for Chattanooga, Urbana, University of Illinois Press, 1994. Pour le front Est, voir Gordon H. Rhea, The Battle of the Wilderness, May 5-6, 1864, Baton Rouge, Louisiana State University Press, 1994); Albert Castel, Decision in the West : The Atlanta Campaign of 1864, Lawrence, University of Kansas Press, 1992 et Joseph T. Glatthaar, The March to the Sea and Beyond: Sherman's Troops in the Savannah and South Carolina Campaigns, New York, New York University Press, 1985.

16. Sur les événements de la période marquant la fin de la Confédération et l'assassinat de Lincoln, voir Jay Winik, April 1865: The Month That Saved America, New York, Harper Collins, 2001. 
chefs-d'œuvre oratoires de la langue anglaise, avec une conclusion vibrante sur une guerre engagée pour qu' "un gouvernement du peuple, par le peuple et pour le peuple ne périsse de cette terre». Son insistance sur le principe d'égalité comme fondateur des États-Unis, sa préférence pour la Déclaration d'indépendance par rapport à la Constitution (qui n'utilise jamais le mot "égal») transformèrent la vision des Américains sur leur nation ${ }^{17}$. De même, son deuxième discours inaugural, prononcé quand il était déjà évident que le Sud allait perdre et promettant «la charité pour tous et aucune malveillance pour quiconque» était d'une magnanimité sincère. Sa capacité à diriger la nation pendant l'épisode le plus traumatisant et le plus éprouvant de son histoire révèle un homme d'État de tout premier ordre. De plus, après avoir mis la question de l'émancipation sur un second plan, il finit par agir. Mais sa réputation actuelle reste prisonnière du mythe. L'opinion sur Lincoln avant son assassinat (en particulier, celle des abolitionnistes, frustrés de son manque d'engagement à la fin de la guerre pour la justice au profit des Noirs américains) était en général différente de celle qui prévalut après sa mort. Lincoln était également d'une nature impitoyable, - trait de caractère méconnu -, qu'il s'agisse d'emprisonner les dizaines de milliers de personnes qui avaient protesté contre la guerre ou de supprimer des journaux qui le critiquaient. De plus, comme toutes les guerres, celle-ci fut gagnée par la force des armes et non par les talents oratoires, et le Nord était beaucoup plus puissant que le Sud. Enfin, même avec son génie politique, Lincoln fut tout aussi surpris que les autres par la force du sentiment sécessionniste sudiste et par le temps qu'il fallut pour conclure victorieusement la guerre. Les historiens devraient se concentrer désormais sur l'homme et non sur le mythe ${ }^{18}$.

Même si nous opposons les entités politiques de la Confédération et de l'Union ou les sociétés du Nord et du Sud, il existait pendant la guerre des divisions sérieuses au sein de chaque camp. Ceci est illustré par l'élection présidentielle de novembre 1864 au cours de laquelle Lincoln brigua un second mandat. L'élection se tenait uniquement dans les États du Nord et il était évident à cette date que le Sud allait perdre. Le président en exercice aurait donc dû gagner avec une écrasante majorité. Cependant, le candidat démocrate (et favorable à l'esclavage) George $\mathrm{B}$. McClellan réussit à rassem-

17. Garry Wills, Lincoln at Gettysburg: The Words that Remade America, New York, Simon and Schuster, 1992.

18. Il est dit que seuls Jésus Christ et William Shakespeare dépassent Lincoln par la quantité d'ouvrages en anglais qui leur sont consacrés. Que ce soit le cas ou non, Lincoln est le sujet de nombreux écrits. Les deux meilleures biographies sont de Richard Carwardine, Lincoln, Harlow, Longman, 2003 et de David Herbert Donald, Lincoln, New York, Simon \& Schuster, 1995. Une étude plus ancienne mais encore classique est de James Randall, Lincoln the President, 4 volumes, New York, Dodd and Mead, 1945-1955. Pour une opinion excessivement critique mais intéressante sur le président, voir Thomas J. Lorenzo, The Real Lincoln: A New Look at Abraham Lincoln, His Agenda and an Unnecessary War, New York, Random House, 2002. Pour l'image de Lincoln dans la culture populaire et la mémoire américaine, voir Andrew Ferguson, Land of Lincoln : Adventures in Abe's America, New York, Atlantic Monthly Press, 2007. 
bler $44 \%$ des suffrages (bien qu'il eût beaucoup moins de succès au sein du collège électoral ${ }^{19}$ ). Entre-temps, l'introduction de la conscription en 1863 fut très impopulaire dans l'Union et occasionna des émeutes importantes et violentes à New York, qui purent être réprimées uniquement par les troupes et l'artillerie. La classe sociale était un facteur déterminant pour la conscription. Les hommes riches pouvaient éviter l'enrôlement en payant 300 dollars - une somme difficilement accessible aux ouvriers. Par ailleurs, les opposants à la guerre, appelés "Copperheads» (du nom d'un serpent venimeux) pouvaient s'attendre à un traitement sévère. Le candidat démocrate pour le poste de gouverneur de l'Ohio, Clement Vallandigham, un opposant à la guerre, fut d'abord condamné à la prison à vie par une cour militaire, avant d'être complètement banni des États-Unis. Cependant, malgré ou à cause de ces efforts, la plupart des nordistes soutenaient d'une manière ou d'une autre l'effort de guerre; le nationalisme américain du XIX siècle s'avéra suffisant pour unir la société dans la perspective d'une guerre longue et difficile ${ }^{20}$.

De l'autre côté, le nationalisme confédéré ne s'avéra pas aussi solide. Les divisions étaient encore plus marquées au Sud qu'au Nord, en particulier lorsque la guerre prit un tour défavorable. Une économie basée sur l'exportation a comme inconvénient de n'avoir aucun recours lorsqu'elle est coupée de ses marchés - ce qui était le cas de la Confédération à la suite du blocus de l'Union. L'inflation (de 10\% par mois en 1863), le stockage et la spéculation sur les produits essentiels posaient sérieusement problème, ainsi que les émeutes pour la nourriture lorsque les produits de base du Sud vinrent à manquer. Les divisions furent exacerbées par le fait que les planteurs pouvaient éviter la conscription sous prétexte de surveiller les esclaves, et par le fait que les esclaves (contrairement aux produits de luxe) n'étaient pas taxés. De plus, quelles que furent les divisions politiques du Nord, celles du Sud étaient plus profondes. Davis se brouilla avec son vice-président qui le dénonça publiquement, et la violence régnait souvent

19. L'élection du président des États-Unis se fait en effet au suffrage indirect : les électeurs votent pour des grands électeurs qui, assemblés en un collège électoral, élisent le président. Les grands électeurs d'un État sont élus en bloc : le candidat qui a la majorité des voix dans un État y obtient la totalité des grands électeurs. Cela rend théoriquement et pratiquement possible l'élection d'un président minoritaire en voix (note de la $R H 19$ ).

20. Sur le sujet des libertés civiques et la situation générale dans les États du Nord voir Heather Cox Richardson, The Greatest Nation on Earth : Republican Economic Policies During the Civil War Cambridge, Harvard University Press, 1997; Catherine Clinton et Nina Silber, eds., Divided Houses : Gender and the Civil War, New York, Oxford University Press, 1992; Mark J. Neely, The Fate of Liberty: Abraham Lincoln and Civil Liberties, New York, Oxford University Press, 1991; Iver Bernstein, The New York City Draft Riots: Their Significance for American Society and Politics in the Age of the Civil War, New York, Oxford University Press, 1990; Phillip S. Paludan, A Peoples' Contest : The Union and the Civil War, 1861-1865, New York, Harper \& Row, 1988; Joel H. Sibley, The Respectable Minority : The Democratic Party in the Civil War Era, 1860-1868, New York, Norton, 1977; Frank L. Clement, Limits of Dissent: Clement L. Vallandigham and the Civil War, Lexington, University of Kentucky, 1970; et George Frederickson, The Inner Civil War : Northern Intellectuals and the Crisis of Disunion, New York, Harper \& Row, 1965. 
lors des débats du Congrès de la Confédération. La théorie sur les droits des États s'avéra être un handicap pour l'effort de guerre sudiste lorsque plusieurs États refusèrent de coopérer avec le gouvernement confédéré à des moments cruciaux de la guerre, pour l'utilisation des hommes ou du matériel. En fin de compte, l'autorité du gouvernement confédéré s'avéra bien moins assise que celle de l'Union. Certains historiens ont même invoqué ces divisions comme expliquant la défaite du Sud ${ }^{21}$.

Cependant, aucune autre autorité ne fut plus faible que celle des propriétaires d'esclaves, et aucun autre groupe sudiste ne fut, bien évidemment moins loyal à la Confédération que la population d'esclaves, qui n'attendit pas l'arrivée des armées de l'Union pour fuir la servitude : au moins un demi-million d'esclaves (sur une population de trois millions et demi) tirèrent profit de la guerre pour s'échapper avant l'intervention des armées de l'Union. Les esclaves commencèrent à s'enfuir bien avant l'entrée en vigueur de la Proclamation de l'émancipation mais, par la suite, le nombre de fugitifs augmenta fortement. Quels que furent les coûts humains du conflit, les esclaves en furent indéniablement les bénéficiaires. Dans les États du Nord, la population noire majoritairement libre était en fait plus divisée sur la guerre. L'omission initiale par Lincoln de l'esclavage comme objectif de guerre impressionna peu. Beaucoup pensèrent que si la préservation des États-Unis impliquait une union avec les propriétaires d'esclaves, la dissolution était préférable. Le racisme ordinaire du gouvernement fédéral, et notamment le refus d'accepter des volontaires noirs dans l'armée était également offensante. Des émeutes contre les Noirs avaient régulièrement lieu dans certaines parties du Nord, celles de New York étant dirigées tout autant contre les Noirs que contre le gouvernement fédéral. Néanmoins, étant donné ses besoins en hommes, le Nord commença à recruter des Noirs, dans des régiments ségrégués, en août 1862. À la fin de la guerre, les Noirs représentaient dix pour cent des troupes de l'Union. $\mathrm{Si}$, jusque dans les années 1960, l'importance de la contribution des Noirs américains à la victoire de l'Union fut largement ignorée (quand ils ne furent pas dépeints comme attendant passivement d'être libérés), les études plus récentes ont largement contribué à rectifier cette négligence ${ }^{22}$.

21. Sur les politiques sudistes et la situation dans les États sudistes, voir Anne S. Rubin, A Shattered Nation: The Rise and Fall of the Confederacy, 1861-1868, Chapel Hill : University of North Carolina Press, 2005; Stephen V. Ash, When the Yankees Came : Conflict and Chaos in the Occupied South, 18611865, Chapel Hill, University of North Carolina Press, 1995; Daniel E. Sutherland, Seasons of War : The Ordeal of a Confederate Community, 1861-1865, New York, Free Press, 1995; Douglas B. Ball, Financial Failure and Confederate Defeat, Urbana, University of Illinois Press, 1991; George C. Rable, Civil Wars: Women and the Crisis of Southern Nationalism, Urbana, University of Illinois Press, 1991; Wayne K. Durrill, War of Another Kind: A Southern Community in the Great Rebellion, New York: Oxford University Press, 1990; Drew Gilpin Faust, The Creation of Confederate Nationalism : Ideology and Identity in the Civil War South, Baton Rouge, Louisiana State University Press, 1988; Emory M. Thomas, The Confederate Nation, 1861-1865, New York, Harper \& Row, 1979.

22. Voir Noah Andre Trudeau, Like Men of War: Black Troops in the Civil War, 1862-1865, Boston, Little and Brown, 1998; William W Freehling, The Reintegration of American History : Slavery and the 
La portée internationale de la guerre de Sécession, en particulier par rapport à l'Atlantique, est un élément encore négligé par la plupart des historiens sérieux. Les études réalisées à ce sujet sont en majorité franchement inadéquates, essayant de faire rentrer de force l'histoire européenne dans un moule américain inadapté. La plus grande erreur est la perception traditionnelle qui divise le sentiment européen en deux : d'un côté, les libéraux qui soutenaient la démocratie américaine et de l'autre, les conservateurs qui étaient en faveur de l'esclavage sudiste. La langue est souvent un élément du problème : trop d'études sont ou ont été écrites par des auteurs qui ne connaissaient que l'anglais comme langue de travail (mais bizarrement, certains ouvrages sur les opinions britanniques sur la guerre sont parmi les pires), ce qui veut dire que ces études reposent fortement sur les rapports consulaires américains, qui de manière évidente, ont leurs limites. Dans ce contexte, les informations présentées ci-après requièrent des études supplémentaires. Cette guerre ne passa pas inaperçue auprès des Européens et suscita des opinions variées. Le tsar Alexandre II et Bismarck soutenaient tous les deux le Nord. Les Russes voyaient les ÉtatsUnis comme un contrepoids essentiel à la Grande-Bretagne et à la France (la Russie avait récemment combattu contre ces pays lors de la guerre de Crimée). Face à la rébellion de la Pologne en 1863-1865, la Russie soutint davantage l'Union. La Prusse considérait également les États-Unis comme un contrepoids utile, mais, engagée dans un processus d'unification des États germaniques en un seul empire, elle avait également des bonnes raisons d'approuver l'usage de la force par Lincoln pour le maintien de l'Union. La Suisse, qui vécut sa propre guerre civile en 1847 , n'était pas non plus inclinée à soutenir la sécession sudiste. Giuseppe Garibaldi, à qui Lincoln proposa un poste de général, soutenait le Nord à cause de l'esclavage. La Grande-Bretagne et la France étaient cependant les deux pays déterminants, car leur intervention aux côtés du Sud aurait certainement assuré l'indépendance de la Confédération ${ }^{23}$.

Même si ces deux nations discutaient entre elles des possibilités de médiation dans la guerre et de reconnaissance diplomatique de la Confédération, les relations embrouillées qu'elles entretenaient ne favorisaient aucun accord possible. Le régime de Napoléon III avait établi une sorte

Civil War, New York, Oxford University Press, 1994; Louis Gerteis, From Contraband to Freedman : Federal Policy Towards Southern Blacks, 1861-1865, Westport, Connecticut : Greenwood Press, 1973; Forrest G. Wood, Black Scare : The Racist Response to Emancipation and Reconstruction, Berkeley, University of California Press, 1968; James M. McPherson, The Negro's Civil War: How American Negroes Felt and Acted During the Civil War, New York, Pantheon Books, 1965 et The Struggle for Equality: Abolitionists and the Negro in the Civil War, Princeton, (New Jersey), Princeton University Press, 1964.

23. Voir Harold Hyman (ed.), Heard Around the World: The Impact Abroad of the Civil War, New York, Knopf, 1969 et B. B. Sideman et L. Friedman (eds.), Europe Looks at the Civil War, New York, Orion Press, 1960. Ces deux ouvrages doivent être traités avec circonspection. Une étude utile qui examine, entre autres, l'impact international de la guerre de Sécession est Christopher A. Bayly, titre en anglais, traduction française, La Naissance du monde moderne, 1780-1914, Ivry-sur-Seine, Éditions de l'Atelier, 2006. 
d'État satellite au Mexique avec l'empereur Ferdinand Maximilian, tandis que la Grande-Bretagne se préoccupait de la sécurité de ses colonies canadiennes. Les deux pays avaient souffert de la pénurie de coton - produit essentiel pour une partie de leurs économies respectives. En fin de compte, la Grande-Bretagne avait moins de raisons d'intervenir et, sans la coopération britannique, la France ne voulait pas agir. L'offre de Napoléon III d'une médiation conjointe fut bien accueillie par Lord John Russell, le ministre britannique aux affaires étrangères, mais à l'exception de celui de William Gladstone, le chancelier de l'Echiquier, il ne put obtenir le soutien du reste du cabinet, et notamment, celui, crucial, du Premier ministre, Lord Palmerston. Palmerston était résolu à éviter la guerre avec les États-Unis et ne voyait aucun avantage à la proposition française. Il aurait également pu subir la pression de la reine Victoria, qui soutenait le Nord. Il est clair qu'aucun de ces gouvernements ne subissait de contrainte en faveur de l'Union de la part de l'opinion populaire. Certains critiques libéraux de Palmerston et de Napoléon III soutenaient le gouvernement de Lincoln, mais les études récentes ont montré qu'ils ne représentaient pas un point de vue majoritaire, même dans leur propre classe politique. Effectivement, il y eut des signes d'une colère populaire et radicale dans les deux pays contre le Nord, en réponse aux sentiments anglophobes et francophobes des Américains (le nationalisme n'était pas un phénomène mineur aux États-Unis). Mais en fin de compte, ni les Britanniques ni les Français ne voulaient rentrer en guerre contre l'Union. Le seul moment où le conflit faillit inclure d'autres belligérants fut lors de l'incident du Trent, lorsque l'Union intercepta un navire britannique dans les eaux internationales et fit débarquer quatre agents confédérés. Cette atteinte à leur neutralité outragea les Britanniques, mais le gouvernement de Palmerston se contenta d'envoyer un ultimatum. Face à l'éventualité d'un soutien français, voire russe, de la position britannique, Washington jugea prudent de relâcher les hommes. En fin de compte, malgré les spéculations fiévreuses sur une intervention européenne dans la guerre, cette éventualité ne fut qu'une chimère. Pour la majorité des Européens, cette guerre était américaine et non européenne. En effet, la rébellion en Pologne (1863-1865) et la guerre prusso-danoise (1864) préoccupèrent davantage la majorité des pays européens (et leurs habitants) ${ }^{24}$.

24. L'étude la plus juste d'un point de vue historique sur le sujet est David Paul Crook, The North, the South and the Powers, New York, Wiley, 1974. Voir également Charles M. Hubbard, The Burden of Confederate Diplomacy, Knoxville, University of Tennessee Press, 2000. Sur la France et la guerre de Sécession, voir : George M. Blackburn, French Newspaper Opinion on the American Civil War, London, Greenwood Press, 1997; Daniel B. Carroll, Henri Mercier and the American Civil War, Princeton (New Jersey), Princeton University Press, 1971; Thomas A. Sancton, «The Myth of French Worker Support for the North in the American Civil War ", French Historical Review, volume 2, n 3, 1962 et Serge Garonsky, French Liberal Opposition and the American Civil War, New York, Humanities Press, 1968. Sur la Grande-Bretagne et la guerre de Sécession, voir Philip Myers, Caution and Co-operation: The American Civil War in British-American Relations, Kent (Ohio), Kent State University Press, à paraître; Frank J. Merli, The Alabama, British Neutrality and the American Civil War, edited and introduced by David M. Fahey, Bloomington, Indiana University Press, 2004; Duncan Andrew Campbell, English 
Il est clair que l'absence d'intervention étrangère de son côté condamna la Confédération. Malgré les nombreux débats sur les raisons de l'échec du Sud, ou sur celles de la victoire du Nord, la bonne réponse se trouve déjà sous la plume de Mme de Sévigné : «La Providence est toujours du côté des grands bataillons». Sans compter les esclaves (qui ne soutenaient pas la Confédération), le Sud était dépassé en nombre d'habitants dans un rapport de 1 pour 4. Le Nord disposait d'une capacité de production industrielle dix fois supérieure à celle du Sud, et produisait $97 \%$ des armes à feu, $94 \%$ du saumon de fonte et $90 \%$ des bottes et des chaussures du pays. En résumé, une nation industrialisée et quatre fois plus peuplée écrasa une économie agraire sous-développée qui ne disposait que du quart des ressources humaines. Il est vrai que l'Union devait conquérir la Confédération, alors que le Sud n'avait pas besoin de dominer le Nord, juste de le forcer à accepter que le prix de la réunification était simplement trop élevé. Dans une guerre limitée, le Sud aurait pu gagner son indépendance. Cependant, dans ce qui s'avéra être pratiquement une guerre totale, impliquant la conscription de masse et l'implication de l'économie dans l'effort de guerre, la Confédération était condamnée. Certes, le Nord dut surmonter des divisions internes et accepter de nombreuses pertes humaines pendant quatre longues années, mais au vu des exemples des pays européens pendant les guerres napoléoniennes et la Première Guerre mondiale, ce n'était pas une exception. La vraie question est de savoir comment le Sud put tenir aussi longtemps, compte tenu de toutes ses faiblesses. La meilleure réponse semble être sa capacité à avoir identifié les meilleurs généraux, tels que Robert E. Lee et Thomas "Stonewall» Jackson avant que le Nord ne trouve Ulysses S. Grant et William Tecumseh Sherman, ainsi que d'avoir mené une guerre défensive. Ce fut aussi un ennemi déterminé : au moins un quart des hommes blancs en âge de porter les armes périrent dans le conflit. Comme le dit Grant en faisant l'inventaire des corps jeunes et âgés de ses ennemis après l'une des dernières batailles : «Ils ont dépouillé le berceau et la tombe». Les sudistes n'étaient pas moins engagés pour leur nouvelle nation que le Nord pour leur Union. Malheureusement pour le Sud, l'engagement seul ne suffit pas ${ }^{25}$.

Public Opinion and the American Civil War, Woodbridge (Suffolk) Royal Historical Society, 2003 et Howard Jones, Union in Peril: The Crisis over British Intervention in the Civil War, Chapel Hill, University of North Carolina Press, 1992. L'auteur voudrait remercier Philip Myers de lui avoir permis de lire son manuscrit.

25. Sur les raisons de la défaite du Sud, voir Gary Gallagher, The Confederate War: How Popular Will, Nationalism, and Military Strategy Could Not Stave Off Defeat, Cambridge (Massachusetts), Harvard University Press, 1997; Gabor S. Boritt (ed.), Why the Confederacy Lost, New York, Oxford University Press, 1992; R. E. Beringer, H. Hattaway, A. Jones et W. N. Still, Why the South Lost the Civil War, Athens, University of Georgia Press, 1986 et David H. Donald (ed.), Why the North Won the Civil War, Baton Rouge, Louisiana State University Press, 1960. 
Suite à l'effondrement de la Confédération, les États-Unis se demandèrent comment les États vaincus allaient être politiquement réintégrés dans l'Union. Entre 1865 et 1877, - période qu'on appelle communément la «Reconstruction»-, les États-Unis eurent la double tâche de réunifier politiquement la nation et de fournir au moins une justice minimale pour les esclaves nouvellement libérés. Peu de sudistes étaient préparés à accepter les Noirs comme leurs égaux sur le plan politique ou social. D'un autre côté, et de manière compréhensible, ces derniers ne pouvaient se contenter que d'une citoyenneté complète, avec tous les droits et les privilèges assimilés. En fin de compte, malgré un Congrès dominé par les Républicains radicaux, qui vota une procédure de destitution (impeachment) du successeur de Lincoln, le président Andrew Johnson, accusé d'être trop clément envers les sudistes blancs tout en ne faisant pas grand-chose pour les anciens esclaves, le besoin de regagner la loyauté de la population blanche l'emporta sur la nécessité d'accorder aux Noirs leurs droits politiques complets. Après le retrait des dernières troupes fédérales en 1877 , les États du sud, avec une souveraineté intérieure retrouvée, introduisirent la ségrégation et privèrent les Noirs progressivement du droit de vote (entrainant un exode hors des États du Sud). Cette situation ne devait pas être sérieusement contestée avant le mouvement des droits civiques des années 1950 et $1960^{26}$.

En ce qui concerne les conséquences générales de la guerre, le Sud devint essentiellement un prolongement colonial du reste des États-Unis sur le plan économique. Le niveau de vie dont il jouissait dans les années 1860 ne devait pas être atteint à nouveau avant 1950. Le Sud devait rester chroniquement sous-développé. Pour le reste de la nation, avec la réhabilitation de l'Union et l'augmentation importante des pouvoirs du gouvernement fédéral, les États-Unis entrèrent dans leur seconde révolution industrielle, en partie grâce aux matières premières du Sud des États-Unis : à terme, la guerre de Sécession devait ainsi contribuer à faire du pays la première puissance mondiale. Ce sont les circonstances que nous connaissons actuellement. Aujourd'hui, les États du Sud, qui ont connu un développement économique rapide depuis la Deuxième guerre mondiale, ont également une influence de plus en plus importante sur le développement économique des États-Unis. Ce processus a conduit certains à diagnostiquer une «méridionalisation» des États-Unis. On a récemment beaucoup dit que l'actuel tournant à droite de la société américaine serait dû à cette montée $\mathrm{du}$ Sud, plus conservateur que le reste du pays : pareille conviction montre bien à quel point l'opposition entre Nord et Sud demeure une ligne de fracture majeure dans la manière dont la société américaine contemporaine

26. Sur la Reconstruction, voir Avery. O. Craven, Reconstruction : Ending of the Civil War, New York, Holt, Rinehart and Winston, 1969 et Eric Foner, Reconstruction : America's Unfinished Revolution, 1863-1877, New York, Harper and Row, 1988. Voir également C. Vann Woodward, The Strange Career of Jim Crow, $3^{\mathrm{e}}$ édition révisée, Oxford, Oxford University Press, 2002. 
se représente à elle-même. Si tel est le cas, cette guerre semble avoir laissé un héritage impossible à effacer ${ }^{27}$.

\section{Duncan Andrew Campbell enseigne l'histoire des Etats-Unis à l'Université de Swansea (Pays de Galles)}

Traduction de l'anglais par Fanja Andriamialisoa (revue par Judith Lyon-Caen et Fabrice Bensimon)

27. Sur les changements dans les États du Nord après la guerre, voir Jack Beatty, Age of Betrayal: The Triumph of Money in America, 1865-1900, New York, Knopf, 2007. Sur le Sud d'après-guerre, voir Dewey W. Grantham, The South in Modern America : A Region at Odds, New York, Harper Collins, 1995. Sur la méridionalisation de l'Amérique, voir Peter Applebome, Dixie Rising: How the South is Shaping American Values, Politics and Culture, New York, Random House, 1996 et Tony Horwitz, Confederates in the Attic: Dispatches from the Unfinished Civil War, New York, Vintage, 1998. 